\title{
Falsche und ungerechtfertigte Unterstellungen gefährden zukünftige Verhandlungen
}

\author{
An einer Pressekonferenz und in einer Medienmitteilung vom 26. Oktober hat \\ santésuisse tendenziös behauptet, dass selbstdispensierende Ärztinnen und Ärzte \\ sowohl über den Einzelleistungstarif TARMED als auch über die Marge Vergütungen \\ für die Medikamente erhalten. Auf diese falsche Unterstellung hat die Taskforce \\ nDMA der FMH (FMH, KKA, MFE, APA) mit dem untenstehenden offenen Brief an \\ Christoffel Brändli, den Verwaltungsratspräsidenten von santésuisse, reagiert; die- \\ ser Brief wurde auch in verschiedenen Medien aufgenommen.
}

\section{Sehr geehrter Herr Präsident}

Mit grossem Befremden und Unverständnis haben wir das santésuisse-Communiqué vom 26. Oktober 2012 sowie die Medienberichte zum Thema Schweizer Medikamenten-Margen zur Kenntnis genommen. Die santésuisse kann selbstredend Studien in Auftrag geben sowie die Öffentlichkeit über deren Ergebnisse informieren.

Bezüglich der Kommentierung der Ergebnisse sowie der Ableitung von politischen Handlungsrichtlinien erwarten wir aber, dass sie sich an bekannte Fakten hält. Bei den Verhandlungen zu einer margenunabhängigen Medikamentenabgabe haben wir der santésuisse/tarifsuisse im Detail und aufgrund einer statistischen Analyse, zahlenbasiert aufgezeigt, dass selbstdispensierende Ärzte für die Medikamentenabgabe keine zusätzlichen TARMED-Positionen verrechnen!

Die santésuisse verbreitet nun wider besseres Wissens und in täuschender Absicht die Botschaft, dass Ärzte sowohl über den Einzelleistungstarif TARMED wie auch über die Marge Vergütungen für die Medikamentenabgabe erhalten. Diese tendenziöse Aussage ist im aktuellen Kontext der Verhandlungen, die wieder aufgenommen werden, nicht akzeptabel. Sie ist nachweislich falsch, entbehrt jeder wissenschaftlichen und statistischen Grundlage, stellt die gesamte Ärzteschaft unter Verdacht, schafft ein politisches Neidklima und torpediert die jüngst in den Spitzengesprächen vereinbarte Wiederannäherung.

Pikanterweise wird auch die von der santésuisse mit den Apothekern vereinbarte Leistungsorientierte Abgabe (LOA), die im Apothekenkanal zusätzlich zu den Margen erhoben wird und Medikamente massgeblich verteuert, weder kritisiert noch erwähnt. Im Gegenteil, den Apotheken sollen sogar noch weitere medizinische Grundversorgeraufgaben übertragen werden, obschon diesen hierfür die Ausbildung, die Qualifikation sowie das nötige Fachwissen fehlt.

Die santésuisse muss sich damit den Vorwurf gefallen lassen, dass diese Berichterstattung gezielt und bewusst unter Missbrauch der öffentlichen Medien eingesetzt wird, um öffentlichen Druck auf Verhandlungsergebnisse auszuüben.

Aufgrund dieser Kommunikation, die wir als direkten politischen Angriff und Vertrauensbruch zwischen Verhandlungspartnern werten, sehen wir die zukünftigen Verhandlungen äusserst gefährdet. Wir erwarten eine klare und zahlenbasierte Stellungnahme und Erklärung zu diesen aus unserer Sicht ungerechtfertigten Unterstellungen. Ausserdem ist für uns nach wie vor unklar, wer nun beim Dachverband der Versicherer zuständig ist für die Verhandlungen einer margenunabhängigen Abgeltung für die ärztliche Leistung der Medikamentenabgabe. Liegt die Zuständigkeit nun bei der «santésuisse» oder bei der Tochtergesellschaft «tarifsuisse»?

Freundliche Grüsse

Dr. med. Jacques de Haller, Präsident der FMH, Dr. med. Ernst Gähler, Vize-Präsident der FMH

Cc: Dr. Christoph Q. Meier, Direktor santésuisse, Stefan Schena, Verwaltungsratspräsident tarifsuisse AG, Verena Nold, Direktorin tarifsuisse AG 